\title{
Niedrigdosis-CT genügt zur Appendizitis-Diagnose
}

\section{Die Diagnose einer akuten Appen- dizitis gelingt auch mittels Niedrig- dosis-Computertomografie mit aus- reichender Sicherheit, sodass es nicht zu einer Erhöhung der nega- tiven Appendektomierate kommt.}

- Zumindest in den USA gilt die Computertomografie des Abdomens heute als bevorzugt verwendete Untersuchungsmethode für die Diagnose einer akuten Appendizitis. Nachdem es sich um eine häufige Erkrankung mit hoher Inzidenz im Kindesalter handelt, gibt allerdings die dabei auftretende Strahlenbelastung zu Besorgnis Anlass.

In einer einfach verblindeten NichtUnterlegenheitsstudie in einem südkoreanischen Krankenhaus wurden 891 Patienten im Alter zwischen 15 und 44 Jahren randomisiert entweder mittels Niedrigdosis-CT oder StandarddosisCT des Abdomens untersucht. In der Niedrigdosisgruppe betrug die mediane Strahlendosis, ausgedrückt als DosisLängen Produkt, $116 \mathrm{mGy} \mathrm{x} \mathrm{cm}$, in der Standarddosis- Gruppe $521 \mathrm{mGy} \mathrm{x} \mathrm{cm}$. Primäres Studienziel war der Prozentsatz negativer Appendektomien, wobei die Nicht-Unterlegenheitsgrenze bei 5,5\% festgelegt wurde. Sekundäre End-

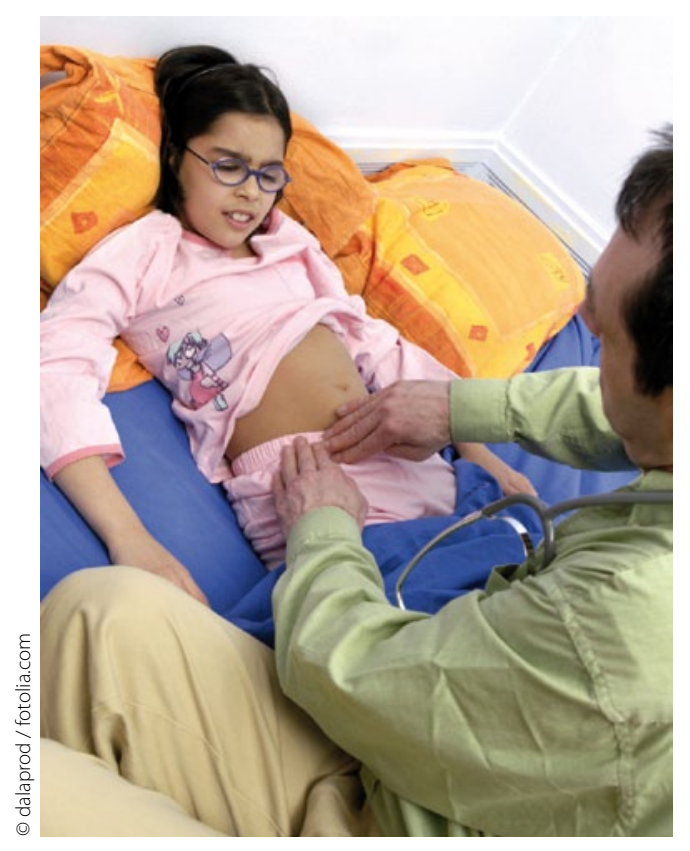

Appendizitis: Diagnose mit Fingerspitzengefühl oder doch lieber mit CT?

punkte beinhalteten die Perforation und den Anteil der Patienten mit Appendizitisverdacht, die eine zusätzliche Bildgebung benötigten.

Die negative Appendektomierate, d. h. der Anteil von Würmern, die keine Entzündungszeichen aufwiesen, betrug in der Niedrigdosis- CT-Gruppe 3,5\% (sechs von 172 Patienten), in der Standarddosis-CT-Gruppe $3,2 \%$ (sechs von 186 Patienten). Die beiden Gruppen unterschieden sich zwar nicht signifikant hinsichtlich der Perforationsraten ( $26,5 \%$ vs. $23,3 \%$ ), allerdings erscheint diese Rate zumindest für deutsche Verhältnisse sehr hoch.

Die Patienten aus der Niedrigdosisgruppe benötigten zwar selten, aber doch doppelt so häufig weitere bildgebende Verfahren, um die Diagnose für die Indikationsstellung zur Operation ausreichend zu sichern (3,2 vs.1,6\%). Bei 166 von 438 Patienten $(37,9 \%)$ der Niedrigdosisgruppe bestätigte der Pathologe die Appendizitis, in der Standarddosisgruppe war dies bei 180 von 441 Patienten der Fall (40,8\%). Somit war der Anteil der Patienten mit tatsächlich vorhandener, pathologisch-anatomisch verifizierter Appendizitis in beiden Gruppen fast gleich groß. 249 bzw. 246 Patienten beider Gruppen konnten ohne Operation nachhause entlassen werden.

\section{Kommentar}

Während sich Generationen von Medizinstudenten durch die Tücken von McBurney und Lanz kämpfen und einen Peritonimus von einer muskulären Abwehrspannung unterscheiden lernen mussten, schickt man Patienten heute bei unklaren Schmerzen im Abdomen rasch zur Computertomografie. Schrittmacher sind auch auf diesem Gebiet die USA mit ihrer stark legalistisch ausgerichteten Defensivmedizin, wo die Mehrzahl der Patienten mit Appendizitisverdacht mittlerweile eine CT bekommt. Mit der massenhaften „Bestrahlung" der Bevölkerung wächst allerdings auch die Sorge vor der
Induktion von Malignomem. Wie sich dieses Risiko auswirkt, werden wir vielleicht erst in 30-40 Jahren erfahren. In der Studie beeindruckt auf alle Fälle die geringe negative Laparotomierate, die in Deutschland noch wesentlich höher ist. Dagegen erstaunt der große Anteil von Patienten mit Perforation. Möglicherweise dauert es in Südkorea doch wesentlich länger als in Deutschland bis Patienten mit Beschwerden von einem Arzt gesehen werden. Die Autoren betonen, dass unter ihren Patienten nur ganz wenige mit Adipositas waren. Insofern erscheint zweifelhaft, ob die guten Ergebnisse mit der Niedrigdosis-CT auf die im Durchschnitt dickeren Patienten in westlichen Ländern übertragbar sind. Von den klinischen Befunden, dem Urteil der untersuchenden Ärzte oder ähnlichen antiquierten Dingen ist gar nicht mehr die Rede. Wir sind auf dem Weg zur berührungsfreien Medizin.

H. S. FÜESSL =

\footnotetext{
- K. Kim et al.

(Korres.: kholeemail@gmail.com): Low-dose abdominal CT for evaluating suspected appendicitis. New Engl. J. Med. 366 (2012) 1596-1605
} 